ISSN 2075-9827 e-ISSN 2313-0210

Carpathian Math. Publ. 2020, 12 (1), 148-156

doi:10.15330/cmp.12.1.148-156

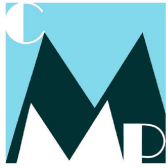

http://www.journals.pnu.edu.ua/index.php/cmp

Карпатські матем. публ. 2020, Т.12, №1, С.148-156

YANCHENKO S.YA.

\title{
APPROXIMATION OF THE NIKOL'SKII-BESOV FUNCTIONAL CLASSES BY ENTIRE FUNCTIONS OF A SPECIAL FORM
}

\begin{abstract}
We establish the exact-order estimates for the approximation of functions from the Nikol'skiiBesov classes $S_{1, \theta}^{r} B\left(\mathbb{R}^{d}\right), d \geq 1$, by entire functions of exponential type with some restrictions for their spectrum. The error of the approximation is estimated in the metric of the Lebesgue space $L_{\infty}\left(\mathbb{R}^{d}\right)$.

Key words and phrases: Nikol'skii-Besov classes, entire function of exponential type, Fourier transform.
\end{abstract}

Institute of Mathematics, National Academy of Sciences of Ukraine, 3 Tereschenkivska str., 01024, Kyiv, Ukraine

E-mail: yan.sergiyegmail.com

\section{INTRODUCTION}

In the paper, we continue to study the approximative characteristics of the Nikol'skii-Besov classes $S_{p, \theta}^{r} B\left(\mathbb{R}^{d}\right)$ of functions with a dominant mixed derivative in the Lebesgue spaces (see $[4,17,18,21,23,25])$. We have established the order estimates of the best approximation of functions from these classes by entire functions of exponential type with a spectrum focused on the Lebesgue sets whose measure does not exceed $M$.

The spaces $S_{p, \theta}^{r} B\left(\mathbb{R}^{d}\right)$ were first considered by S. M. Nikol'skii [8] for $\theta=\infty$ (in this case also one can $\left.S_{p, \infty}^{r} B\left(\mathbb{R}^{d}\right) \equiv S_{p}^{r} H\left(\mathbb{R}^{d}\right)\right)$ and T. I. Amanov [1] for $1 \leq \theta<\infty$. In the classical form, the definition of these functional spaces was formulated by S. M. Nikol'skii and T. I. Amanov through mixed multiple differences and mixed multiple modules of continuity of functions. Here, the definition of the Nikol'skii-Besov spaces $S_{p, \theta}^{r} B\left(\mathbb{R}^{d}\right)$ is presented through so-called decomposition representation of the norm of elements from these spaces. Note that decomposition representation and corresponding rationing of the Nikol'skii-Besov spaces were first obtained by S. M. Nikol'skii and P. I. Lizorkin [5]. As it turned out, this decomposition norm of functions plays a key role in the studies of different approximative characteristics of the function classes. This representation is based on the application of the Fourier transform that can be defined using generalized functions (see, e.g., [2, Ch. 11], [6], [15, Ch. 2]).

$\mathrm{y} \triangle \mathrm{K} 517.51$

2010 Mathematics Subject Classification: 41A30, 41A50, 41A63.

This work was partially supported by President's of Ukraine grant for competitive projects (№ F84/177-2019) and Grant of the NAS of Ukraine to research groups of young scientists of the NAS of Ukraine for research by priority development areas of science and technology, project №04-02/2019. 


\section{DEFINITION OF CLASSES OF FUNCTIONS AND APPROXIMATIVE CHARACTERISTICS}

Let $\mathbb{R}^{d}$ be the $d$-dimensional Euclidean space with the elements $x=\left(x_{1}, \ldots, x_{d}\right)$ and $(x, y):=x_{1} y_{1}+\cdots+x_{d} y_{d}$. Denote by $L_{q}\left(\mathbb{R}^{d}\right), 1 \leq q \leq \infty$, the space of all functions $f(\boldsymbol{x})=$ $f\left(x_{1}, \ldots, x_{d}\right)$ measurable on $\mathbb{R}^{d}$ with the finite norm

$$
\|f\|_{q}:=\left(\int_{\mathbb{R}^{d}}|f(\boldsymbol{x})|^{q} d \boldsymbol{x}\right)^{\frac{1}{q}}, 1 \leq q<\infty, \quad \text { and } \quad\|f\|_{\infty}:=\underset{x \in \mathbb{R}^{d}}{\operatorname{ess} \sup }|f(\boldsymbol{x})| .
$$

Let $S=S\left(\mathbb{R}^{d}\right)$ be the Schwarz space of test complex-valued functions $\varphi$ infinitely differentiable on $\mathbb{R}^{d}$ and decreasing at infinity together with their derivatives faster than any power of the function $\left(x_{1}^{2}+\ldots+x_{d}^{2}\right)^{-\frac{1}{2}}$, considered in the appropriate topology. Let $S^{\prime}$ denote the space of linear continuous functionals on $S$. The elements of the space $S^{\prime}$ are generalized functions. If $f \in S^{\prime}$, then $\langle f, \varphi\rangle$ denotes the value of a functional $f$ on the test function $\varphi \in S$. Denote by $\mathfrak{F} \varphi$ and $\mathfrak{F}^{-1} \varphi$ the Fourier transform and the inverse Fourier transform of functions $\varphi$ from the spaces $S$ and $S^{\prime}$.

For any continuous function $\varphi$ on $\mathbb{R}^{d}$, the closure of the set of all points $x \in \mathbb{R}^{d}$ such that $\varphi(\boldsymbol{x}) \neq 0$ is called the support of the function $\varphi$ and denoted by supp $\varphi$.

The generalized function $f$ vanishes in an open set $G$ when $\langle f, \varphi\rangle=0$ for all $\varphi \in S$ and supp $\varphi \subset G$. The union of all neighborhoods, where $f$ is equal to zero, is an open set and called the null set of the generalized function $f$. It is denoted by $G_{f}$. The complement of the largest open set $G_{f}$ to $\mathbb{R}^{d}$ is called the support of the generalized function $f$, i.e., $\operatorname{supp} f$ equals to $\bar{G}_{f}$, it is a closed set.

According to the formula

$$
\langle f, \varphi\rangle=\int_{\mathbb{R}^{d}} f(\boldsymbol{x}) \varphi(\boldsymbol{x}) d \boldsymbol{x}, \quad \varphi \in S,
$$

each function $f \in L_{p}\left(\mathbb{R}^{d}\right), 1 \leq p \leq \infty$, defines a linear continuous functional on $S$ and, therefore, is an element of $S^{\prime}$ in this sense. Hence, the Fourier transform of a function $f \in L_{p}\left(\mathbb{R}^{d}\right)$, $1 \leq p \leq \infty$, can be regarded as the Fourier transform of the generalized function (1).

Further, let $K_{m}(t)=\int_{\mathbb{R}} k_{m}(\lambda) e^{-2 \pi i \lambda t} d \lambda, m \in \mathbb{Z}_{+}, K_{-1}:=0$, where

$$
k_{m}(\lambda)=\left\{\begin{array}{ll}
1, & |\lambda|<2^{m-1}, \\
2\left(1-\frac{|\lambda|}{2^{m}}\right), & 2^{m-1} \leq|\lambda| \leq 2^{m}, \\
0, & |\lambda|>2^{m},
\end{array} \quad k_{0}(\lambda)= \begin{cases}1-|\lambda|, & 0 \leq|\lambda| \leq 1, \\
0, & |\lambda|>1 .\end{cases}\right.
$$

For any vector $s=\left(s_{1}, \ldots, s_{d}\right), s_{j} \in \mathbb{Z}_{+}, j=\overline{1, d}$, we define

$$
\begin{gathered}
A_{\boldsymbol{s}}^{*}(\boldsymbol{x})=\prod_{j=1}^{d}\left(K_{s_{j}}\left(x_{j}\right)-K_{s_{j}-1}\left(x_{j}\right)\right), \\
A_{\boldsymbol{s}}^{*}(f, \boldsymbol{x})=f(\boldsymbol{x}) * A_{\boldsymbol{s}}^{*}(\boldsymbol{x})=\int_{\mathbb{R}^{d}} f(\boldsymbol{y}) A_{\boldsymbol{s}}^{*}(\boldsymbol{x}-\boldsymbol{y}) d \boldsymbol{y} .
\end{gathered}
$$

Also, for all $s \in \mathbb{Z}_{+}^{d}$, consider the sets

$$
Q_{2^{s}}^{*}=\left\{\lambda=\left(\lambda_{1}, \ldots, \lambda_{d}\right): \eta\left(s_{j}\right) 2^{s_{j}-1} \leq\left|\lambda_{j}\right|<2^{s_{j}}, \lambda_{j} \in \mathbb{R}, j=\overline{1, d}\right\},
$$


where $\eta(0)=0$ and $\eta(t)=1, t>0$ (respectively, $Q_{2^{s}}^{*}$ for $d=1$ ).

The following statement is true.

Lemma 1 (see example [4]). Let $1 \leq p \leq \infty$, then for any $f \in L_{p}\left(\mathbb{R}^{d}\right)$, we have

$$
f(x)=\sum_{s} A_{s}^{*}(f, x)
$$

and $\operatorname{supp} \mathfrak{F} A_{s}(f, x) \subseteq Q_{2^{s}}^{*}$

Note that $A_{s}^{*}(f, x)$ is the analog of the de la Vallée Poussin block of sum of periodic function of several variables (see example [14]).

In the accepted notation, the spaces $S_{p, \theta}^{r} B\left(\mathbb{R}^{d}\right), 1 \leq p, \theta \leq \infty, \boldsymbol{r}>0$, can be defined as follows (see, e.g., $[4,16])$ :

$$
S_{p, \theta}^{r} B\left(\mathbb{R}^{d}\right):=\left\{f \in L_{p}\left(\mathbb{R}^{d}\right):\|f\|_{S_{p, \theta}^{r} B}<\infty\right\},
$$

where for $1 \leq \theta<\infty$,

$$
\|f\|_{S_{p, \theta}^{r} B\left(\mathbb{R}^{d}\right)} \asymp\left(\sum_{s \geq 0} 2^{(s, r) \theta}\left\|A_{s}^{*}(f, \cdot)\right\|_{p}^{\theta}\right)^{\frac{1}{\theta}}
$$

and for $\theta=\infty$,

$$
\|f\|_{S_{p, \infty}^{r} B\left(\mathbb{R}^{d}\right)}:=\|f\|_{S_{p}^{r} H\left(\mathbb{R}^{d}\right)} \asymp \sup _{s \geq 0} 2^{(s, r)}\left\|A_{s}^{*}(f, \cdot)\right\|_{p} .
$$

Here and below, for positive quantities $a$ and $b$, the notation $a \asymp b$ means that there exist positive constants $C_{1}$ and $C_{2}$ that do not depend on an essential parameter in the values $a$ and $b$ (e.g., $C_{1}$ and $C_{2}$ in the expressions (2) and (3) do not depend on the function $f$ ) such that $C_{1} a \leq b$ (in this case, we write $a \ll b$ ) and $C_{2} a \geq b$ (in this case, we write $a \gg b$ ). In the present paper, all constants $C_{i}, i=1,2, \ldots$, depend only on the parameters contained in the definition of the function class, the metric in which we estimate the error of approximation, and the dimension of the space $\mathbb{R}^{d}$. Moreover, for the vectors $\boldsymbol{a}=\left(a_{1}, \ldots, a_{d}\right)$ and $\boldsymbol{b}=\left(b_{1}, \ldots, b_{d}\right)$, the inequalities of the type $\boldsymbol{a} \leq \boldsymbol{b}(\boldsymbol{a}>\boldsymbol{b})$ are understood in the coordinate-wise: $a_{j} \leq b_{j}\left(a_{j}>b_{j}\right)$, $j=\overline{1, d}$. We also use $\boldsymbol{t} \geq 0(\boldsymbol{t}>0)$ if $t_{j} \geq 0\left(t_{j}>0\right), j=\overline{1, d}$, and $\boldsymbol{a} \neq \boldsymbol{b}$ if $a_{i} \neq b_{i}$ at least for one $i, i=\overline{1, d}$.

In what follows, we use the notations $S_{p, \theta}^{r} B$ and $S_{p}^{r} H\left(S_{p, \theta}^{r} B\right.$ and $S_{p}^{r} H$ for $\left.d=1\right)$ instead of $S_{p, \theta}^{r} B\left(\mathbb{R}^{d}\right)$ and $S_{p}^{r} H\left(\mathbb{R}^{d}\right)$ respectively. We also assume that the coordinates of the vector $\boldsymbol{r}=\left(r_{1}, \ldots, r_{d}\right)$ are ordered as follows $0<r_{1}=r_{2}=\cdots=r_{v}<r_{v+1} \leq \cdots \leq r_{d}$. The vector $r=\left(r_{1}, \ldots, r_{d}\right)$ is associated with the vector $\gamma=\left(\gamma_{1}, \ldots, \gamma_{d}\right), \gamma_{j}=r_{j} / r_{1}, j=\overline{1, d}$, and the vector $\gamma$ is, in turn, associated, with the vector $\gamma^{\prime}$, where $\gamma_{j}^{\prime}=\gamma_{j}$, if $j=\overline{1, v}$ and $1<\gamma_{j}^{\prime}<\gamma_{j}$, $j=\overline{v+1, d}$.

In addition, in the case $1<p<\infty$, the norm of functions from the spaces $S_{p, \theta}^{r} B\left(\mathbb{R}^{d}\right)$ can be defined in another form. Let $A \subset \mathbb{R}^{d}$ be a measurable set. Denote by $\chi_{A}$ a characteristic function of the set $A$ and for $f \in L_{p}\left(\mathbb{R}^{d}\right)$, set $\delta_{s}^{*}(f, x)=\mathfrak{F}^{-1}\left(\chi_{Q_{2 s}^{*}} \cdot \mathfrak{F} f\right)$. The spaces $S_{p, \theta}^{r} B$, $1<p<\infty, 1 \leq \theta \leq \infty, \boldsymbol{r}>0$, can be defined as follows [5]

$$
S_{p, \theta}^{r} B:=\left\{f \in L_{p}\left(\mathbb{R}^{d}\right):\|f\|_{S_{p, \theta}^{r} B}<\infty\right\}
$$


where

$$
\|f\|_{S_{p, \theta}^{r} B} \asymp\left(\sum_{s \geq 0} 2^{(s, r) \theta}\left\|\delta_{s}^{*}(f, \cdot)\right\|_{p}^{\theta}\right)^{\frac{1}{\theta}}
$$

for $1 \leq \theta<\infty$ and

$$
\|f\|_{S_{p}^{r} H} \asymp \sup _{s \geq 0} 2^{(s, r)}\left\|\delta_{s}^{*}(f, \cdot)\right\|_{p} .
$$

The class $S_{p, \theta}^{r} B$ is defined as a set of functions $f \in L_{p}\left(\mathbb{R}^{d}\right)$ such that $\|f\|_{S_{p, \theta}^{r} B} \leq 1$. We preserve the same notations for the classes $S_{p, \theta}^{r} B$ as for the spaces $S_{p, \theta}^{r} B$.

As can be seen from (2)-(5), for any $f \in S_{p, \theta}^{r} B, 1<p<\infty$, the following relation holds:

$$
\left\|\delta_{s}^{*}(f, \cdot)\right\|_{p} \asymp\left\|A_{s}^{*}(f, \cdot)\right\|_{p} .
$$

Now we consider the approximative characteristics of the classes $S_{p, \theta}^{r} B$.

Let $\mathcal{L} \subset \mathbb{Z}_{+}^{d}$ be a finite set, $\mathfrak{M}:=\mathfrak{M}(\mathcal{L})=\bigcup_{s \in \mathcal{L}} Q_{2^{*}}^{*}$. For any $f \in L_{q}\left(\mathbb{R}^{d}\right), 1 \leq q \leq \infty$, we put

$$
S_{\mathfrak{M}}(f, x):=S_{\mathfrak{M}(\mathcal{L})}(f, x)=\sum_{s \in \mathcal{L}} \delta_{s}^{*}(f, x) .
$$

Since supp $S_{\mathfrak{M}}(f, x) \subseteq \mathfrak{M}$, then $S_{\mathfrak{M}}(f, x)$ is an entire function of the space $L_{q}\left(\mathbb{R}^{d}\right)$.

For $f \in L_{q}\left(\mathbb{R}^{d}\right)$ and $S_{p, \theta}^{r} B\left(\mathbb{R}^{d}\right) \subset L_{q}\left(\mathbb{R}^{d}\right)$, consider the following approximative characteristic

$$
e_{M}^{\mathfrak{F}}(f)_{q}:=\inf _{\mathcal{L}: \operatorname{mes} \mathfrak{M}(\mathcal{L}) \leq M}\left\|f(\cdot)-S_{\mathfrak{M}(\mathcal{L})}(f, \cdot)\right\|_{q}
$$

and

$$
e_{M}^{\mathfrak{F}}\left(S_{p, \theta}^{r} B\right)_{q}:=\sup _{f \in S_{p, \theta}^{r} B} e_{M}^{\mathfrak{F}}(f)_{q}
$$

\section{ApproximAtion OF FUNCTIONS FROM CLASSES $S_{1, \theta}^{r} B\left(\mathbb{R}^{d}\right)$ BY ENTIRE FUNCTIONS}

The following statements are true.

Theorem 1. Let $r>1,1 \leq \theta \leq \infty$ and $d=1$. Then the following relation holds:

$$
e_{M}^{\mathfrak{F}}\left(S_{1, \theta}^{r} B(\mathbb{R})\right)_{\infty} \asymp M^{-r+1} .
$$

Theorem 2. Let $r_{1}>1,1 \leq \theta \leq \infty$. Then for $d \geq 2$ the following relation holds:

$$
e_{M}^{\mathfrak{F}}\left(S_{1, \theta}^{r} B\left(\mathbb{R}^{d}\right)\right)_{\infty} \asymp\left(M^{-1} \log ^{\nu-1} M\right)^{r_{1}-1}\left(\log ^{\nu-1} M\right)^{1-\frac{1}{\theta}} .
$$

The results of Theorems 1 and 2 are also new for Nikol'skii classes $S_{1}^{r} H\left(\mathbb{R}^{d}\right), d \geq 1$.

Let us note that in Theorem 1 , the estimate $e_{M}^{\mathfrak{F}}\left(S_{1, \theta}^{r} B(\mathbb{R})\right)_{\infty}$ does not depend on the parameter $\theta$ unlike to the corresponding estimate in the case $d \geq 2$ (Theorem 2).

Before proving the main results, we formulate auxiliary theorem.

Theorem 3 ([1]). Let $1 \leq p, \theta \leq \infty, 1 \leq p \leq q \leq \infty$ and we have a vector $\rho$ such that $\rho_{j}=r_{j}-\left(\frac{1}{p}-\frac{1}{q}\right)>0, j=\overline{1, d}$. If $f \in S_{p, \theta}^{r} B\left(\mathbb{R}^{d}\right)$, then $f \in S_{q, \theta}^{\rho} B\left(\mathbb{R}^{d}\right)$ and

$$
\|f\|_{S_{q, \theta}^{\rho} B\left(\mathbb{R}^{d}\right)} \ll\|f\|_{S_{p, \theta}^{r} B\left(\mathbb{R}^{d}\right)} .
$$


Proof of Theorem 1. Since $r>1$, then by virtue of Theorem 3, there exists a number $\rho$, $\rho=r-1>0$, such that for any function $f \in S_{1, \theta}^{r} B(\mathbb{R})$, we have $f \in S_{\infty, \theta}^{\rho} B(\mathbb{R}) \subset L_{\infty}(\mathbb{R})$.

First, we will get the upper estimate in (7). Recall now the definition of another approximative characteristic used in the proof of the results. For $s \in \mathbb{Z}_{+}^{d}$, define the set $Q_{n}^{\gamma}$ as follows

$$
Q_{n}^{\gamma}=\bigcup_{(s, \gamma) \leq n} Q_{2^{s}}^{*}
$$

where $n \in \mathbb{N}$. The set $Q_{n}^{\gamma}$ is called a stepwise hyperbolic cross and, moreover, mes $Q_{n}^{\gamma} \asymp$ $2^{n} n^{d-1}$ (see, e.g., [5]), where mes $Q_{n}^{\gamma}$ is the Lebesgue measure of the set $Q_{n}^{\gamma}$.

For $f \in L_{q}\left(\mathbb{R}^{d}\right), 1 \leq q \leq \infty$, we set

$$
S_{Q_{n}^{\gamma}}(f, x)=\sum_{(s, \gamma) \leq n} \delta_{s}^{*}(f, x), x \in \mathbb{R}^{d}
$$

and denote

$$
\mathcal{E}_{Q_{n}^{\gamma}}(f)_{q}=\left\|f(\cdot)-S_{Q_{n}^{\gamma}}(f, \cdot)\right\|_{q} \quad \text { and } \quad \mathcal{E}_{Q_{n}^{\gamma}}\left(S_{p, \theta}^{r} B\right)_{q}=\sup _{f \in S_{p, \theta}^{r} B} \mathcal{E}_{Q_{n}^{\gamma}}(f)_{q} .
$$

We now specificating the definition of the quantity $\mathcal{E}_{Q_{n}^{\gamma}}(f)_{q}$ in the one-dimensional case.

For $d=1$, each of the sets $Q_{2^{s}}^{*}$ is a union of the half intervals $\left(-2^{s},-2^{s-1}\right]$ and $\left[2^{s-1}, 2^{s}\right)$, $s \in \mathbb{Z}_{+}$, with the corresponding modification at $s=0$. Then the stepwise hyperbolic cross degenerates into the interval $\left(-2^{n}, 2^{n}\right)$, as the union of sets $Q_{2^{s}}^{*}$ for all $s \leq n, s \in \mathbb{Z}_{+}$, namely $Q_{n}:=Q_{n}^{\gamma}=\bigcup_{s \leq n} Q_{2^{s}}^{*}$. In addition we have $\left|Q_{n}\right| \asymp 2^{n}$, where $\left|Q_{n}\right|$ denotes the length of the interval.

The definition of (9) for $f \in L_{q}(\mathbb{R}), 1 \leq q \leq \infty$, can be rewritten as follows

$$
\mathcal{E}_{Q_{n}}(f)_{q}=\left\|f(\cdot)-S_{Q_{n}}(f, \cdot)\right\|_{q}, \quad \mathcal{E}_{Q_{n}}\left(S_{p, \theta}^{r} B\right)_{q}=\sup _{f \in S_{p, \theta}^{r} B} \mathcal{E}_{Q_{n}}(f)_{q},
$$

where

$$
S_{Q_{n}}(f, x)=\sum_{s \leq n} \delta_{s}^{*}(f, x)
$$

From the definition of the approximative characteristics (6) and (9), it follows that the following relation holds in the case when mes $Q_{n}^{\gamma} \asymp$ mes $\mathfrak{M}$

$$
e_{M}^{\mathfrak{F}}\left(S_{p, \theta}^{r} B\right)_{q} \ll \mathcal{E}_{Q_{n}^{\gamma}}\left(S_{p, \theta}^{r} B\right)_{q} .
$$

The following statement is true.

Theorem 4 ([25]). Let $r_{1}>1,1 \leq \theta \leq \infty$. Then the following relation holds:

$$
\mathcal{E}_{Q_{n}^{\gamma}}\left(S_{1, \theta}^{r} B\right)_{\infty} \asymp 2^{-n\left(r_{1}-1\right)} n^{(v-1)\left(1-\frac{1}{\theta}\right)} .
$$

In the case $d=1$, the estimate (11) can be written as follows

$$
\mathcal{E}_{Q_{n}}\left(S_{1, \theta}^{r} B\right)_{\infty} \asymp 2^{-n(r-1)} .
$$

For a given $M$, we choose a number $n \in \mathbb{N}$ such that $\left|Q_{n}\right| \leq M<\left|Q_{n+1}\right|$, i.e. $M \asymp 2^{n}$. Taking into account (10), from relation (12) we get the upper estimate in (7)

$$
e_{M}^{\mathfrak{F}}\left(S_{1, \theta}^{r} B\right)_{\infty} \ll \mathcal{E}_{Q_{n}}\left(S_{1, \theta}^{r} B\right)_{\infty} \asymp 2^{-n(r-1)} \asymp M^{-r+1} .
$$


To obtain the lower estimate in (7), for any $n \in \mathbb{N}$, consider the function

$$
f_{1}(x)=C_{3} 2^{-n r} A_{n}^{*}(x), \quad C_{3}>0,
$$

that is, the function $f_{1}$ consists of one "block" $A_{n}^{*}(x)$.

We give some auxiliary statements.

Lemma 2 ([25]). Let $1 \leq p<\infty$, then the estimate

$$
\left\|A_{s}^{*}(\cdot)\right\|_{p} \asymp 2^{\|s\|_{1}\left(1-\frac{1}{p}\right)}
$$

holds, where $\|s\|_{1}=s_{1}+\ldots+s_{d}, s_{j} \in \mathbb{Z}_{+}, j=\overline{1, d}$.

Lemma 3 ([25]). The following relation

$$
\left\|A_{s}^{*}(\cdot)\right\|_{\infty} \asymp 2^{\|s\|_{1}}
$$

holds, where $\|s\|_{1}=s_{1}+\ldots+s_{d}, s_{j} \in \mathbb{Z}_{+}, j=\overline{1, d}$.

According to Lemma 2, we have $\left\|A_{s}^{*}(\cdot)\right\|_{1} \asymp C_{4}$. Then for $1 \leq \theta<\infty$,

$$
\left\|f_{1}\right\|_{S_{1, \theta}^{r} B} \asymp\left(\sum_{s} 2^{s r \theta}\left\|A_{s}^{*}\left(f_{1}, \cdot\right)\right\|_{1}^{\theta}\right)^{\frac{1}{\theta}} \asymp\left(2^{n r \theta} 2^{-n r \theta}\right)^{\frac{1}{\theta}}=1
$$

and

$$
\left\|f_{1}\right\|_{S_{1, \infty}^{r}} \asymp \sup _{s} 2^{s r}\left\|A_{s}^{*}\left(f_{1}, \cdot\right)\right\|_{1} \asymp 2^{n r} 2^{-n r}=1 .
$$

So, the function $f_{1}$ belongs to the class $S_{1, \theta}^{r} B$ for all $1 \leq \theta \leq \infty$.

For a given $M$, choosing a number $n \in \mathbb{N}$ such that $\left|\widetilde{Q}_{n}\right| \leq 4 M<\left|\widetilde{Q}_{n+1}\right|$, where $\widetilde{Q}_{n}=Q_{2^{n}}^{*}$, $\left|\widetilde{Q}_{n}\right| \asymp 2^{n}$, and using Lemma 3 , we conclude that

$$
\left\|f_{1}(\cdot)-S_{\mathfrak{M}}\left(f_{1}, \cdot\right)\right\|_{\infty} \geq\left|\left\|f_{1}(\cdot)\right\|_{\infty}-\left\|S_{\mathfrak{M}}\left(f_{1}, \cdot\right)\right\|_{\infty}\right| \gg 2^{-n r}\left(2^{n}-M\right) \gg 2^{-n r} 2^{n} \asymp M^{-r+1} .
$$

The lower estimate is established. Theorem 1 is proved.

Before proving Theorem 2 we note that by Theorem 3 the condition $r_{1}>1$ ensures that there exists a vector $\rho, \rho_{j}=r_{j}-1>0, j=\overline{1, d}$, such that any function $f \in S_{1, \theta}^{r} B\left(\mathbb{R}^{d}\right)$ belongs to the set $S_{\infty, \theta}^{\rho} B\left(\mathbb{R}^{d}\right)$ and therefore $f \in L_{\infty}\left(\mathbb{R}^{d}\right)$. In addition, we can say that for some $1<q_{0}<\infty$, $f \in S_{q_{0}, \theta}^{\rho} B$, where $\rho_{j}=r_{j}-\left(1-\frac{1}{q_{0}}\right)>0, j=\overline{1, d}$.

Proof of Theorem 2. The upper estimate in (8) follows from Theorem 4. Since mes $Q_{n}^{\gamma} \ll$ $2^{n} n^{v-1}$, then for a given $M$, we choose a number $n \in \mathbb{N}$ such that mes $Q_{n}^{\gamma} \leq M<$ mes $Q_{n+1}^{\gamma}$, that is $M \asymp 2^{n} n^{v-1}$. Using relation (11), we have

$$
e_{M}^{\mathfrak{F}}\left(S_{1, \theta}^{r} B\right)_{\infty} \ll 2^{-n\left(r_{1}-1\right)} n^{(v-1)\left(1-\frac{1}{\theta}\right)} \asymp\left(M^{-1} \log ^{\nu-1} M\right)^{r_{1}-1}\left(\log ^{\nu-1} M\right)^{\left(1-\frac{1}{\theta}\right)} .
$$

Passing to establishing the estimate from below in (8), we should note that it is sufficient to obtain it in the case $v=d$.

Let

$$
\Theta(n)=\left\{s=\left(s_{1}, \ldots, s_{d}\right) \in \mathbb{Z}^{d}: s_{1}+\ldots+s_{d}=n\right\} \quad \text { and } \quad \widetilde{Q}_{n}=\bigcup_{s \in \Theta(n)} Q_{2^{s}}^{*}
$$


and mes $\widetilde{Q}_{n} \asymp 2^{n} n^{\nu-1}$.

Unlike the one-dimensional case, we consider the following functions depending on the value of the parameter $\theta$

$$
f_{2}(x)=C_{5} 2^{-n r_{1}} n^{-\frac{d-1}{\theta}} \sum_{s \in \Theta(n)} A_{\boldsymbol{s}}^{*}(\boldsymbol{x}), \quad C_{5}>0,
$$

when $1 \leq \theta<\infty$, and

$$
f_{3}(x)=C_{6} 2^{-n r_{1}} \sum_{s \in \Theta(n)} A_{s}^{*}(x), \quad C_{6}>0
$$

when $\theta=\infty$.

Let us show that the functions $f_{2}$ and $f_{3}$ belong to the classes $S_{1, \theta}^{r} B$ and $S_{1, \infty}^{r} B$ respectively. According to Lemma 2, we have $\left\|A_{s}^{*}(\cdot)\right\|_{1} \asymp C_{7}$. Then

$$
\begin{aligned}
\left\|f_{2}\right\|_{S_{1, \theta}^{r} B} & \asymp\left(\sum_{s \in \Theta(n)} 2^{(s, r) \theta}\left\|A_{s}^{*}\left(f_{2}, \cdot\right)\right\|_{1}^{\theta}\right)^{\frac{1}{\theta}} \asymp 2^{-n r_{1}} n^{-\frac{d-1}{\theta}}\left(\sum_{s \in \Theta(n)} 2^{(s, r) \theta}\left\|A_{s}^{*}(\cdot)\right\|_{1}^{\theta}\right)^{\frac{1}{\theta}} \\
& \asymp 2^{-n r_{1}} n^{-\frac{d-1}{\theta}}\left(\sum_{s \in \Theta(n)} 2^{r_{1}(s, 1) \theta}\right)^{\frac{1}{\theta}} \ll n^{-\frac{d-1}{\theta}}\left(\sum_{s \in \Theta(n)} 1\right)^{\frac{1}{\theta}} \ll 1 .
\end{aligned}
$$

For the function $f_{3}$, the following estimates hold:

$$
\left\|f_{3}\right\|_{S_{1, \infty}^{r}} \asymp \sup _{s \in \Theta(n)} 2^{(s, r)}\left\|A_{s}^{*}\left(f_{3}, \cdot\right)\right\|_{1} \asymp 2^{-n r_{1}} \sup _{s \in \Theta(n)} 2^{(s, r)}\left\|A_{s}^{*}(\cdot)\right\|_{1} \asymp 2^{-n r_{1}} \sup _{(s, 1)=n+1} 2^{(s, r)} \ll 1 .
$$

Further, denote by $\mathcal{L}^{\prime}$ the set of vectors $s$ such that $s \in \Theta(n)$ and the set $\mathfrak{M}=\mathfrak{M}\left(\mathcal{L}^{\prime}\right)=$ $\bigcup_{s \in \mathcal{L}^{\prime}} Q_{2^{s}}^{*}$ satisfies the relation

$$
\text { mes } \widetilde{Q}_{n} \leq 4 M<\text { mes } \widetilde{Q}_{n+1} \text {, }
$$

where $M=M(n)=$ mes $\mathfrak{M}$.

Lemma 4 ([25]). The following relation holds:

$$
\left\|\sum_{(s, 1)=n+1} A_{s}^{*}(\cdot)\right\|_{\infty} \asymp 2^{n} n^{d-1}
$$

Using the Lemmas 3, 4 and relation (13), taking into account that mes $\widetilde{Q}_{n} \asymp 2^{n} n^{v-1}$, we can write

$$
\begin{aligned}
\left\|f_{2}(\cdot)-S_{\mathfrak{M}}\left(f_{2}, \cdot\right)\right\|_{\infty} & \geq\left|\left\|f_{2}(\cdot)\right\|_{\infty}-\left\|S_{\mathfrak{M}}\left(f_{2}, \cdot\right)\right\|_{\infty}\right| \\
& \gg 2^{-n r_{1}} n^{\frac{d-1}{\theta}}\left(2^{n} n^{d-1}-M\right) \gg 2^{-n r_{1}} n^{\frac{d-1}{\theta}} 2^{n} n^{d-1} \\
& =2^{-n\left(r_{1}-1\right)} n^{(d-1)\left(1-\frac{1}{\theta}\right)} \asymp\left(M^{-1} \log ^{d-1} M\right)^{r_{1}-1}\left(\log ^{d-1} M\right)^{\left(1-\frac{1}{\theta}\right)} .
\end{aligned}
$$

Similarly in the case $\theta=\infty$, we get

$$
\left\|f_{3}(\cdot)-S_{\mathfrak{M}}\left(f_{3}, \cdot\right)\right\|_{\infty} \gg\left(M^{-1} \log ^{d-1} M\right)^{r_{1}-1} \log ^{d-1} M .
$$


The lower estimates are established. Theorem 2 is proved.

The exact-order estimates of $e_{M}^{\mathfrak{F}}\left(S_{p, \theta}^{r} B\right)_{q}$ are established in [21] for some other relations between parameters $p, q$ and $\theta$. In this article, we show that there are relations between the parameters $p, q, \theta$ such that the quantities $e_{M}^{\mathfrak{F}}\left(S_{p, \theta}^{r} B\right)_{q}$ and $\mathcal{E}_{Q_{n}^{\gamma}}\left(S_{p, \theta}^{r} B\right)_{q}$ have different orders.

The quantity (6) is a non-periodic analogue of the best orthogonal approximation and the quantity (9) corresponds to the approximation of the stepwise hyperbolic Fourier sum. The main results concerning the approximation of the Nikol'skii-Besov classes of periodic functions with a dominant mixed derivative can be found in monographs V.N. Temlyakov [14], A. S. Romanyuk [11] and D. Dũng, V. N. Temlyakov and T. Ullrich [3].

Currently, the generalizations of the Nikol'skii-Besov classes with the dominant mixed smoothness of periodic and non-periodic functions of many variables are currently being intensively studied, in particular, in the articles $[7,9,10,12,13,19,26]$.

In the one-dimensional case, the Nikol'skii-Besov classes with mixed smoothness $S_{p, \theta}^{r} B\left(\mathbb{R}^{d}\right)$ coincide with isotropic and anisotropic Nikol'skii-Besov classes $B_{p, \theta}^{r}\left(\mathbb{R}^{d}\right)$ and $B_{p, \theta}^{r}\left(\mathbb{R}^{d}\right)$. The exact-order estimates of some approximate characteristics of these classes are established in $[20,22,24]$.

\section{REFERENCES}

[1] Amanov T.I. Representation and embedding theorems for function spaces $S_{p, \theta}^{(r)} B\left(\mathbb{R}_{n}\right)$ and $S_{p, \theta}^{(r) *} B,\left(0 \leq x_{j} \leq 2 \pi\right.$; $j=1, \ldots, n)$. Tr. Mat. Inst. Steklova 1965, 77, 5-34. (in Russian)

[2] Berezansky Yu.M., Sheftel Z.G., Us G.F. Functional analysis. Institute of Matematics of NAS of Ukraine. Vol. 1, Kyiv, 2010.

[3] Dũng D., Temlyakov V.N., Ullrich T. Hyperbolic Cross Approximation. Birkhäuser, Basel, 2018.

[4] Heping W., Yongsheng S. Approximation of functions in $\widetilde{S_{1}^{r} L}, S_{1}^{r} H$ by entire functions. Approx. Theory \& its Appl. 1999, 15 (4), 88-93. doi:10.1007/BF02848673

[5] Lizorkin P.I., Nikol'skii S.M. Function spaces of mixed smoothness from the decomposition point of view. Proc. Steklov Inst. Math. 1990, 187, 163-184. (translation of Tr. Mat. Inst. Steklova 1989, 187, 143-161. (in Russian))

[6] Lizorkin P.I. Generalized Liouville differentiation and the multiplier method in the theory of imbeddings of classes of differentiable functions. Tr. Mat. Inst. Steklova 1969, 105, 89-167. (in Russian)

[7] Myroniuk V.V., Yanchenko S.Ya. Approximation of functions from generalized Nikol'skii-Besov classes by entire functions in Lebesgue spaces. Mat. Stud. 2013, 39 (2), 190-202. (in Ukrainian)

[8] Nikol'skii S.M. Functions with dominant mixed derivative, satisfying a multiple Hölder condition. Sibirsk. Mat. Zh. 1963, 4 (6), 1342-1364. (in Russian)

[9] Pustovoitov N.N. Approximation of multidimensional functions with a given majorant of mixed moduli of continuity . Math. Notes 1999, 65 (1), 89-98. doi:10.1007/BF02675013 (translation of Mat. Zametki 1999, 65 (1), $107-117$. doi:10.4213/mzm1032 (in Russian))

[10] Pustovoitov N.N. The representation and approximation of periodic functions of several variables with a given mixed modulus of continuity. Anal. Math. 1994, 20, 35-48. doi:10.1007/BF01908917 (in Russian)

[11] Romanyuk A.S. Approximative Characteristics of the Classes of Periodic Functions of Many Variables. Proc. of the Institute of Mathematics of the NAS of Ukraine, Kiev, 2012, 93. (in Russian)

[12] Stasyuk S.A. Best approximations and Kolmogorov and trigonometric widths of the classes $B_{p, \theta}^{\Omega}$ of periodic functions of many variables. Ukrainian Math. J. 2004, 56 (11), 1849-1863. doi:10.1007/s11253-005-0155-1 (translation of Ukrain. Mat. Zh. 2004, 56 (11), 1557-1568. (in Ukrainian))

[13] Stasyuk S.A., Yanchenko S.Ya. Approximation of functions from Nikolskii-Besov type classes of generalized mixed smoothness. Anal. Math. 2015, 41 (4), 311-334. doi:10.1007/s10476-015-0305-0 
[14] Temlyakov V.N. Approximation of functions with bounded mixed derivative. Proc. Steklov Inst. Math. 1989, 178, 1-121. (translation of Tr. Mat. Inst. Steklova 1986, 178, 1-112. (in Russian))

[15] Vladimirov V.S. Equations of Mathematical Physics. Nauka, Moscow, 1981. (in Russian)

[16] Wang H. Quadrature formulas for classes of functions with bounded mixed derivative or difference. Sci. China Math. 1997, 40 (5), 449-458. doi:10.1007/BF02896952

[17] Wang H., Yongsheng S. Approximation of multivariate functions with a certain mixed smoothness by entire functions. Northeast. Math. J. 1995, 11 (4), 454-466.

[18] Yanchenko S.Ya. Approximation of functions from the classes $S_{p, \theta}^{r} B$ in the uniform metric. Ukrainian Math. J. 2013, 65 (5), 771-779. doi:10.1007/s11253-013-0813-7 (translation of Ukrain. Mat. Zh. 2010, 62 (8), 698-705. (in Ukrainian))

[19] Yachenko S.Ya. Approximations of classes $B_{p, \theta}^{\Omega}$ of functions of many variables by entire functions in the space $L_{q}\left(\mathbb{R}^{d}\right)$. Ukrainian Math. J. 2010, 62 (1), 136-150. doi:10.1007/s11253-010-0338-2 (translation of Ukrain. Mat. Zh. 2010, 62 (1), 123-135. (in Ukrainian))

[20] Yachenko S.Ya. Approximation of functions from the isotropic Nikol'skii-Besov classes in the uniform and integral metrics. Ukrainian Math. J. 2016, 67 (10), 1599-1610. doi:10.1007/s11253-016-1175-8 (translation of Ukrain. Mat. Zh. 2015, 67 (10), 1423-1433. (in Ukrainian))

[21] Yanchenko S.Ya. Approximation of the classes $S_{p, \theta}^{r} B\left(\mathbb{R}^{d}\right)$ of functions of many variables by entire functions of a special form. Ukrainian Math. J. 2011, 62 (8), 1307-1325. doi:10.1007/s11253-011-0431-1 (translation of Ukrain. Mat. Zh. 2010, 62 (8), 1124-1138. (in Ukrainian))

[22] Yachenko S.Ya. Best approximation of the functions from anisotropic Nikol'skii-Besov classes defined in $\mathbb{R}^{d}$. Ukrainian Math. J. 2018, 70 (4), 661-670. doi:10.1007/s11253-018-1523-y (translation of Ukrain. Mat. Zh. 2018, 70 (4), 574-582. (in Ukrainian))

[23] Yanchenko S.Ya. Estimates for approximative characteristics of classes $S_{p, \theta}^{r} B\left(\mathbb{R}^{d}\right)$ of functions in the uniform metric. $\mathrm{Zb}$. Pr. Inst. Mat. NAN Ukr. Collection of Works "Approximation Theory of Functions and Related Problems", 2013, 10 (1), 328-340. (in Ukrainian).

[24] Yanchenko S.Ya. Order estimates of approximation characteristics of functions from the anisotropic Nikol'skii-Besov classes. J. Math. Sci. (N.Y.) 2018, 234 (1), 98-105. doi:10.1007/s10958-018-3984-9 (translation of Ukr. Mat. Visn. 2017, 14 (4), 595-605. (in Ukrainian))

[25] Yanchenko S.Ya., Radchenko O.Ya. Approximating characteristics of the Nikol'skii-Besov classes $S_{1, \theta}^{r} B\left(\mathbb{R}^{d}\right)$. Ukrainian Math. J. 2020, 71 (10), 1608-1626. doi:10.1007/s11253-020-01734-9 (translation of Ukrain. Mat. Zh. 2019, 71 (10), 1405-1421. (in Ukrainian))

[26] Yongsheng S., Heping W. Representation and approximation of multivariate periodic functions with bounded mixed moduli of smoothness. Proc. Steklov Inst. Math. 1997, 219 (4), 350-371. (translation of Tr. Mat. Inst. Steklova 1997, 219, 356-377. (in Russian))

Received 04.03.2020

Revised 13.05.2020

Янченко С.Я. Наближення класів функцій Нікольського-Бесова иілими функиіями спеціального вигляду // Карпатські матем. публ. - 2020. - Т.12, №1. - С. 148-156.

Одержано точні за порядком оцінки наближення функцій з класів Нікольського-Бєсова $S_{1, \theta}^{r} B\left(\mathbb{R}^{d}\right), d \geq 1$, за допомогою цілих функцій експоненціального типу з певними обмеженнями на їхній спектр. Похибка наближення оцінюється у метриці простору $\Lambda$ ебега $L_{\infty}\left(\mathbb{R}^{d}\right)$.

Ключові слова і фрази: класи Нікольського-Бєсова, ціла функція експоненціального типу, перетворення Фур'є. 